\title{
Characterization of the slow extraction frequency response
}

\author{
M. Pari®, ${ }^{*} \dagger$ F. M. Velotti®, M. A. Fraser®, V. Kain®, and O. Michels \\ CERN, 1211 Geneva 23, Switzerland
}

(Received 17 December 2020; accepted 16 June 2021; published 5 August 2021)

\begin{abstract}
The main physics program of the CERN Super Proton Synchrotron (SPS) is dedicated to the fixed target physics experiments hosted in the North experimental Area (NA). Protons are delivered to the NA via thirdinteger resonant slow extraction over an almost $5 \mathrm{~s}$ flattop. In order to maximize the usable intensity delivered to the experiments, the flux of extracted particles should be kept as constant as possible. This is a very general requirement for fixed target experiments served by synchrotrons. Power supply ripples are a well-known issue in resonant slow extraction, affecting the quality of the spill. A long-standing effort is ongoing at CERN to characterize the SPS slow extraction frequency response to its main power supplies. In this paper, beam dynamics simulations are employed to understand and characterize the process, combined with dedicated beam based measurements.
\end{abstract}

DOI: 10.1103/PhysRevAccelBeams.24.083501

\section{INTRODUCTION}

The CERN Super Proton Synchrotron (SPS) is the last accelerator in the Large Hadron Collider (LHC) injector chain. It has a circumference of about $6.9 \mathrm{~km}$ and can accelerate protons up to $450 \mathrm{GeV}$. Since its construction (terminated in 1976), the SPS has mostly been used for providing beam for fixed target experiments. Currently, up to $4.5 \times 10^{13}$ protons of $400 \mathrm{GeV}$ can be extracted in $4.8 \mathrm{~s}$ to the North experimental Area (NA) fixed target experiments and user facilities, exploiting a slow resonant extraction.

The slow extraction at SPS is a chromatic-based third integer resonant extraction, where the beam is extracted along its momentum distribution by sweeping the horizontal tune of the machine across a $2 / 3$ resonance. In particular, the horizontal tune is swept by acting simultaneously on all the main quadrupoles of the machine during the flattop, and, since recently, together with all other active elements of the SPS [1,2]. The main requirement from the experiments is a constant flux of extracted particles: any perturbation in the temporal structure of the spill could possibly lead to problems in the particle detection process, as increased counting uncertainties or detector saturation. This is true for any frequency component in the spill, from

\footnotetext{
*michelangelo.pari@cern.ch

Also at: Physics Department G. Galilei, Universitá degli Studi di Padova; INFN Sezione di Padova, Via Marzolo 8, 35131 Padova, Italy.

Published by the American Physical Society under the terms of the Creative Commons Attribution 4.0 International license. Further distribution of this work must maintain attribution to the author(s) and the published article's title, journal citation, and DOI.
}

the power supply $50 \mathrm{~Hz}$ harmonics to the $200 \mathrm{MHz}$ radio frequency (rf) structure of the beam. To minimize the effects of the latter, the beam is debunched during extraction. The main power supply currents are the principal responsible for a large part of the harmonics seen on the extracted beam structure. Unfortunately, there is no easy solution to minimize their effect in the outgoing particle flux, in fact this is a topic of decades long studies in many of the slow extraction based accelerators around the world [3-10]. In order to optimize the SPS spill quality, both the macrostructure and microstructure are targeted separately [11]. Currently, an iterative feed-forward algorithm acting on all the main quadrupoles [12] is employed to optimize the macrostructure of the spill to that of a square pulse. This algorithm does not have a significant effect on the power supply ripples because it is mostly sensible to large scale variations of the spill signal. In the past, a real-time feedback system acting on a set of four correcting quadrupoles was being used for the task [13]; this system had to be decommissioned since the variation introduced on the extracted beam trajectory was leading to mis-steering on the targets [12] and consequent loss of useful intensity for the experiments. The ripple content of the spill is targeted by another iterative feed-forward system which uses a set of two servo-quadrupoles to minimize the main four $50 \mathrm{~Hz}$ harmonics measured on the spill by a secondary emission monitor sampled at $2 \mathrm{kHz}$. Both iterative feed-forward methods described here apply the corrections estimated from the measured spill signal at the subsequent slow extraction machine cycle, until the optimum solution is reached. This process can take several machine cycles to converge (usually about ten) and the stability of the reached solution relies upon the reproducibility of the magnets' response and power supply ripples in the SPS. It has been 
observed that a change in machine supercycle modifies the magnets' response to current [11], and also that the phase and amplitude stability of the $50 \mathrm{~Hz}$ harmonics can be subjected to significant changes in a days to weeks basis [14]: both of these facts trigger sudden changes in the spill quality, and call for periodical reiterations of the feedforward correction methods. The regulator feedback loop of the main power converters also attempts to suppress the harmonics components by injecting opposite phase harmonics, but it was observed experimentally that this introduces low frequency noise, which was finally worse for the experiments (the loop gain is high enough only at low frequencies, e.g., $\lesssim 20 \mathrm{~Hz}$ ).

The beam dynamics of the slow extraction process itself acts as a low pass filter to current ripples. This remarkable fact comes from the time-to-extraction distribution of unstable particles in phase space, of which a full theoretical derivation is carried out in [15]. Earlier studies at the SPS, dating back to 1983 , already identified such an effect to be best represented by a second order low pass filter with a time delay [16,17]. These results have been used for the improvement of the previously used spill feedback system. More recent studies at the SPS $[18,19]$ seemed to confirm the past observed low pass filter behavior, and have shown that no significant increase in extraction losses should be observed for realistic amplitudes of focusing quadrupole ripples. An analogous campaign at the J-PARC main ring used the current-to-spill transfer function in order to improve the ripple-correcting feedback system there employed [20]. It has also been proven that the feedback system could be replaced by a promising new approach, in which a real-time estimation of the tune variation made from the measured quadrupole and dipole currents is used to inject a counteracting current signal on some dedicated quadrupoles [21]. This approach could be considered as a real-time feed-forward system, particularly advantageous when the ripples are not stable from one machine cycle to another. Another possible way to improve the spill quality is to increase the smoothing effect of the time intervals distribution of the extracted particles, by acting on the machine parameters (from a frequency point of view, it corresponds to increasing the attenuation of the low pass filter of the extraction process). Recent studies at GSI SIS18 synchrotron have shown how a reduced extraction sextupole strength and beam emittance can help in suppressing the ripples on the extracted spill, due to the increased smearing of the extraction time intervals distribution $[22,23]$. Intuitively, the importance of the extraction sextupole strength on the response to ripples is related to the fact that it defines the speed and acceleration of unstable particles in phase space, and with it the spread of the extraction intervals distribution. This key fact has also been observed at the SPS in a study apt to maximize the response to quadupolar pulses of the machine [24]. Modulation techniques based on the rf are also another actively used and established way to reduce the effect of magnet ripples. For instance, the injection of longitudinal stochastic rf noise, so to generate a diffusive flux of particles to resonance, has been proven to be a ripple-suppressing method since its first applications at the CERN-PS and SPS $[25,26]$. The use of a rf phase displacement technique (empty bucket channeling) allows instead to selectively speed up the particles approaching resonance, reducing the sensitivity to magnet ripples [15,27-29]. Similarly, a simple bunched slow extraction has also been shown to improve the spill quality [22]. While these techniques can offer an advantageous mitigation of the low frequency spectral components $(\lesssim \mathrm{kHz})$, they can introduce higher frequency components due to the use of the rf, or impose impractical constraints for the case of the present SPS slow extraction: for this reason, they will not be covered here. Inspired by the results of the latter techniques [30], a method of injecting a dominant quadrupolar ripple at a frequency higher than the main harmonics and above the cutoff frequency of the low pass filter effect by a definite factor, has been shown to improve the overall spill quality at GSI [31].

In summary, the knowledge of the slow extraction low pass filter effect covers an important role in order to exploit at best the methods of spill quality improvement.

In the present paper, the frequency response of the slow extraction process at the SPS is fully characterized, with the goal to better understand it and how it interplays with the different machine parameters. In Sec. II, different simulation models of the process will be developed, and important aspects of it will be investigated exploiting the developed models. In Sec. III, the data from dedicated measurements at the SPS will be used to validate the simulation models, while in Sec. IV, another validation will be attempted by using operational data from physics runs. Section $\mathrm{V}$ will show how the developed simulation models could be used to look for machine optima in terms of ripple suppression, just by scanning a few key slow extraction parameters. Some considerations on applying the developed models to different machines are drawn in Sec. VI. Finally, Sec. VII will summarize the obtained results, and Sec. VIII will outline the future developments.

\section{MODELING AND SIMULATION OF SLOW EXTRACTION FREQUENCY RESPONSE}

A ripple on the input current of the main magnets will be transferred to the extracted spill according to a certain transfer function, which includes both purely hardware and beam dynamics effects. Overall, the problem will be treated as follows:

$$
i(t)=i_{0}(t)+\Delta i(t) \rightarrow s(t)=s_{0}(t)+\Delta s(t),
$$

where $i(t)$ indicates the input signal, composed by an ideal component $i_{0}$ and a perturbation $\Delta i$; the same holds for the 
spill, $s(t)$. In the typical case $i_{0}$ is a nearly linear ramp and $s_{0}$ a constant, while $\Delta i$ and $\Delta s$ are a combination of discrete harmonics components and continuous noise. The present study will focus on the amplitude of the transfer function $T_{a}$ of the noise signals, defined as

$$
T_{a}(\omega)=\left\|\frac{\widetilde{\Delta s}(\omega)}{\widetilde{\Delta i}(\omega)}\right\|,
$$

where $\widetilde{\Delta s}$ and $\widetilde{\Delta i}$ are the Fourier transforms of $\Delta s$ and $\Delta i$, respectively. Equation (2) represents the full transfer function of the slow extraction process, which is exactly what the model will try to represent. This transfer function is not adimensional, as the units of measurement of the system change from input to output. Moreover, the employed units can depend on the particular model or application being considered. For instance, the input $(\Delta i)$ could be measured in units of Ampere, normalized quadrupole strength $\left(\mathrm{m}^{-2}\right)$, or tune. The absolute value of the output $(\Delta s)$, typically expressed in particles per second, will ultimately depend on the total number of extracted particles. When comparing experimentally obtained transfer functions with simulated ones (or also different simulation models), scaling errors are to be expected, caused by mismatches in the input quantities or calibration constants. For this reason, the units and absolute values of the computed transfer functions can be subjected to changes throughout this work and will often be expressed as arbitrary.

\section{A. Transfer function block model of the SPS}

The slow extraction process comprises a series of different transformations that can be organized in the fashion of a cascaded circuit model, as shown in Fig. 1.

As the SPS slow extraction is a quadrupole driven resonant extraction, and the resonance is on the horizontal plane, the main contributors to the spill quality are the focusing quadrupoles. In particular, the contribution from other active elements, as extraction sextupoles and main dipoles, has been shown in literature to be significantly lower $[18,19,22]$; the same has been verified also for the defocusing quadrupoles by using MADX [32]. For these reasons the input signal for the present work will be assumed to be the current of the main focusing quadrupoles. An example of a measured current signal of the main quadrupoles can be observed in the top left plot of Fig. 1 [corresponding to $i(t)$, in the formalism of Eq. (1)], while the spectrum of its ripple component (i.e., $\|\widetilde{\Delta i}\|$ ) is reported in the bottom left plot. In Fig. 1 (right), a full extracted spill and its spectrum [i.e., respectively $s(t)$ and $\|\widetilde{\Delta s}\|]$ are shown.

The first gray block of Fig. 1 represents the effects of the magnet losses and shielding from the vacuum chamber: the input current is converted in the effective magnetic field which is seen by the beam. This particular block embodies all the hardware effects which cannot be simulated using a tracking code. The contribution of this effect will be evaluated from measurements.

The second and third blocks represent the slow extraction process. They can either be seen as one transformation, or decomposed in two. In the following subsections, details of the modeling of each of these blocks are given in order to characterize the slow extraction frequency response.

It is important to point out that despite the process being represented in the fashion of a cascaded circuit model, no initial assumption of linearity and cascadability of each block has been made.

\section{Magnet strength to spill simulation results}

The second and third gray blocks of Fig. 1 can be simulated together (i.e., enclosing block "Spill/kQ") using

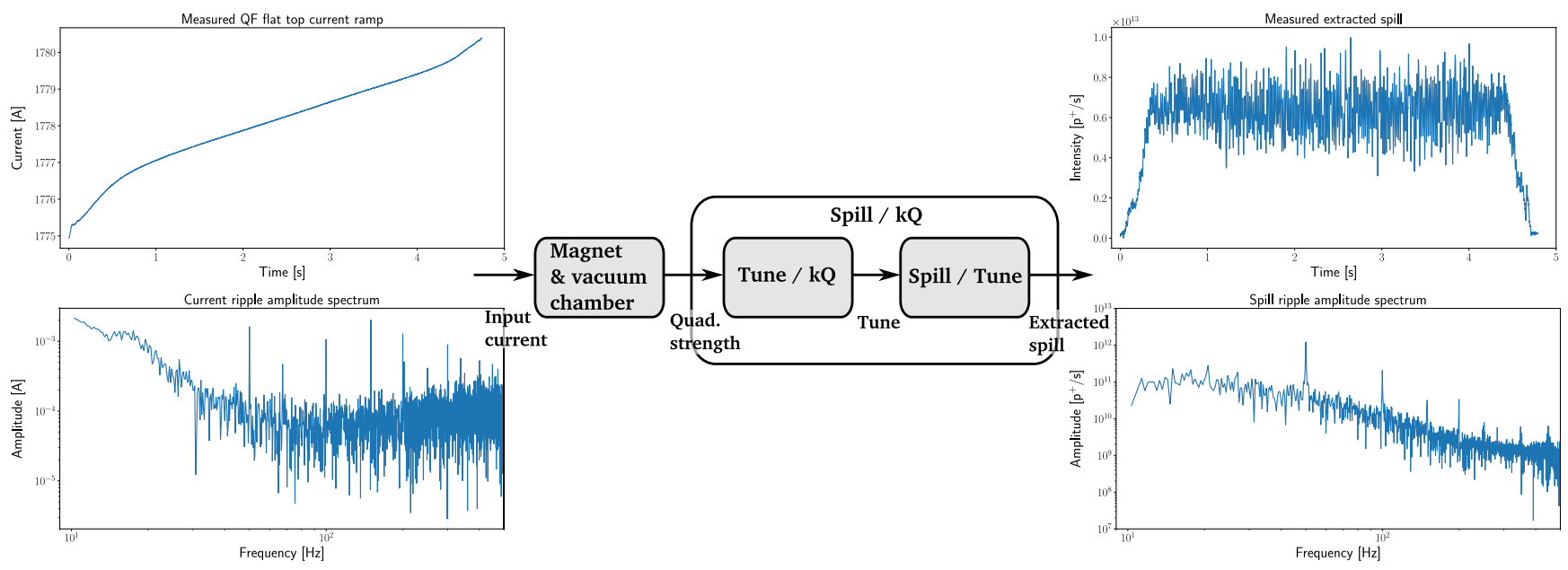

FIG. 1. Schematic model of the slow extraction process in terms of its transfer function blocks. The plots on the left are a typical example of the focusing quadrupole current ramp (top) and the spectrum of its ripple component (bottom). The plots on the right are a typical operational extracted spill (top) and the spectrum of its ripple component (bottom), computed in the fashion of Eq. (1). 
a MADX implementation of the SPS slow extraction [33]. Many studies based on this model have shown impressive agreement with data $[2,34,35]$. In order to build a transfer function of the process, single frequency sinusoidal ripples are injected one at a time in addition to the nominal quadrupole function used to drive the resonance. The amplitude of the corresponding frequency component is then measured on the spill signal, and normalized by the input amplitude. Each ripple has the following expression:

$$
r(t)=A_{K} \cdot \sin (2 \pi f t+\phi),
$$

where $A_{K}$ is the amplitude (in units of the quadrupole strength), $f$ is the frequency (in Hertz) and $\phi$ is a phase shift. Since the focus of the present study is on the amplitude of the transfer function, $\phi$ is assumed to be constant and equal to 0 , without loss of generality. The harmonic analysis of the ripples on the spill are performed subtracting the dc component. In order not to introduce any bias in doing so, a fiducial time interval is selected around the center of the spill, to exclude the two transients at the beginning and end of the extraction. The spectral components of the perturbation are then obtained either by using a fast Fourier transform (FFT) or the numerical analysis of fundamental frequencies (NAFF) algorithm [36,37]. The NAFF algorithm has shown the most accurate results, hence it was chosen as the default method for the following analysis.

Figure 2 shows the transfer functions obtained for different values of the input amplitude, $A_{K}$. Several remarks can be drawn from the result: (i) Below a certain input amplitude $\left(\simeq 2.5 \times 10^{-8} \mathrm{~m}^{-2}\right)$, all the obtained transfer functions are comparable to each other, and no amplitude dependence is observed. Above such an amplitude, a nonlinear behavior emerges, making the transfer function dependent on the input amplitude. (ii) A zero of the transfer

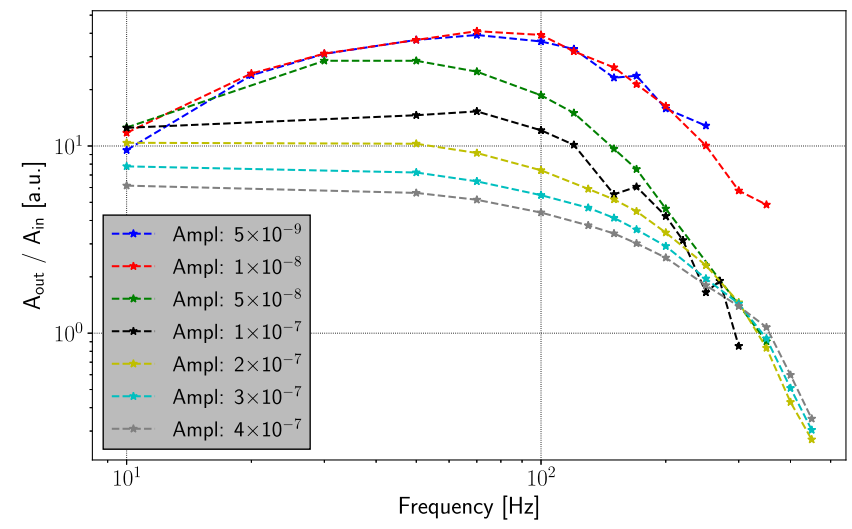

FIG. 2. Simulated fixed amplitude transfer functions for the full extraction process. The injected amplitudes are reported in the legend and are expressed in units of $\mathrm{m}^{-2}$, i.e., normalized quadrupole strength. For reference, the nominal quadrupole strength is of the order of $1.5 \times 10^{-2} \mathrm{~m}^{-2}$. function is present only for the small input amplitudes, for which an initial growth from low to high frequencies can be observed. (iii) All the obtained transfer functions behave as a low pass filter, with a cutoff frequency around $\sim 100 \mathrm{~Hz}$.

The first two points can be readily derived by considering a uniform momentum distribution of the protons, as simulated in the MADX model. In this case, assuming an instantaneous extraction of particles and an ideal momentum extraction, the spill can be approximated by the expression:

$$
\frac{d N(t)}{d t}= \begin{cases}C \frac{d Q(t)}{d t} & \text { if } Q(t)>\left.Q(\tau)\right|_{\tau \in\left[t_{i}, t\right]} \\ 0 & \text { otherwise, }\end{cases}
$$

where $d N / d t$ represents the number of extracted particles per second, $Q$ is the horizontal tune of the machine, $C$ a constant, and $t_{i}$ the start of the extraction. It is possible to justify observation (i) by noticing that Eq. (4) is a linear differential equation only if the tune is a monotonic function. In this case, the equation is that of a derivator circuit, which explains the presence of a zero for small amplitudes. The last observation, which is not foreseen by the ideal model of Eq. (4), is related to the distribution of the extraction times of unstable particles in response to a tune variation (as described in [15]), also referred to as the "interval distribution" of the extracted particles. This behavior is in line with literature, previous studies, and machine observations, as previously detailed in Sec. I.

Even with the simple model of Eq. (4), useful insights can be obtained by considering the case of a linear tune sweep superimposed to a sinusoidal ripple:

$$
Q_{\mathrm{eff}}(t)=\frac{\Delta Q}{\Delta t} t+Q_{i}+A_{Q} \cdot \sin (2 \pi f t),
$$

where $Q_{i}$ is the initial point of the ideal tune ramp, $\Delta Q$ the ideal tune variation, $\Delta t$ is the flattop duration and $A_{Q}$ is the amplitude of the ripple in tune. Imposing monotonicity on $Q_{\text {eff }}(t)$ yields

$$
A_{Q} 2 \pi f \leq\left|\frac{\Delta Q}{\Delta t}\right| .
$$

Using the value of $\Delta Q / \Delta t$ for the SPS in Eq. (6) and converting from tune to quadrupole strength, it is found that $A_{K} \simeq 2.4 \times 10^{-8} \mathrm{~m}^{-2}$ delimits the linear from nonlinear behavior of Eq. (4) for $f=50 \mathrm{~Hz}$ : this is in good agreement with the simulation results [observation (i) and Fig. 2]. To understand the scale of this phenomenon, the latter threshold value corresponds to about $1.6 \mathrm{ppm}$ of the nominal quadruopole strength at resonance, and to about $0.7 \%$ of the total tune variation.

Equation (4), which already allows to identify a source of nonlinear behavior, can also be used to draw some considerations on the time invariance of the system. For 
dominant nonmonotonic slow signals the time invariance is broken by the gradual depletion of different tune regions. The low pass filter effect [i.e., inertia of particles being extracted, not included in Eq. (4)] acts against such depletion, implying time invariance to hold for high enough frequencies $f \gg 1 / \tau$, where $\tau$ is the finite length of the spill. In the particular case of a monotonicity-preserving ripple on top of a linear tune ramp and uniform momentum distribution, the momentum extraction of the SPS implies a fixed presentation of the particles at resonance, and ensures the time invariance of the system (excluding the initial and final transients of the spill). This could also be extended to periodic nonmonotonic ripples with amplitude sufficiently smaller than $\Delta Q$. However, in a more general case of nonuniform momentum distribution, the tune ramp for achieving a uniform macrostructure of the spill could present significant changes in slope, which can break the time invariance. As a worst case scenario, a small-amplitude linear ripple could become nonlinear on different parts of the ramp, intertwining nonlinearity and time invariance. For the case of the SPS, the feed-forward algorithm acting on the spill macro structure leads to the typical ramp seen in the top-left plot of Fig. 1. Thanks to the rf gymnastic, the SPS momentum distribution is not far from a uniform one, and it is possible to verify that the deviations from a linear
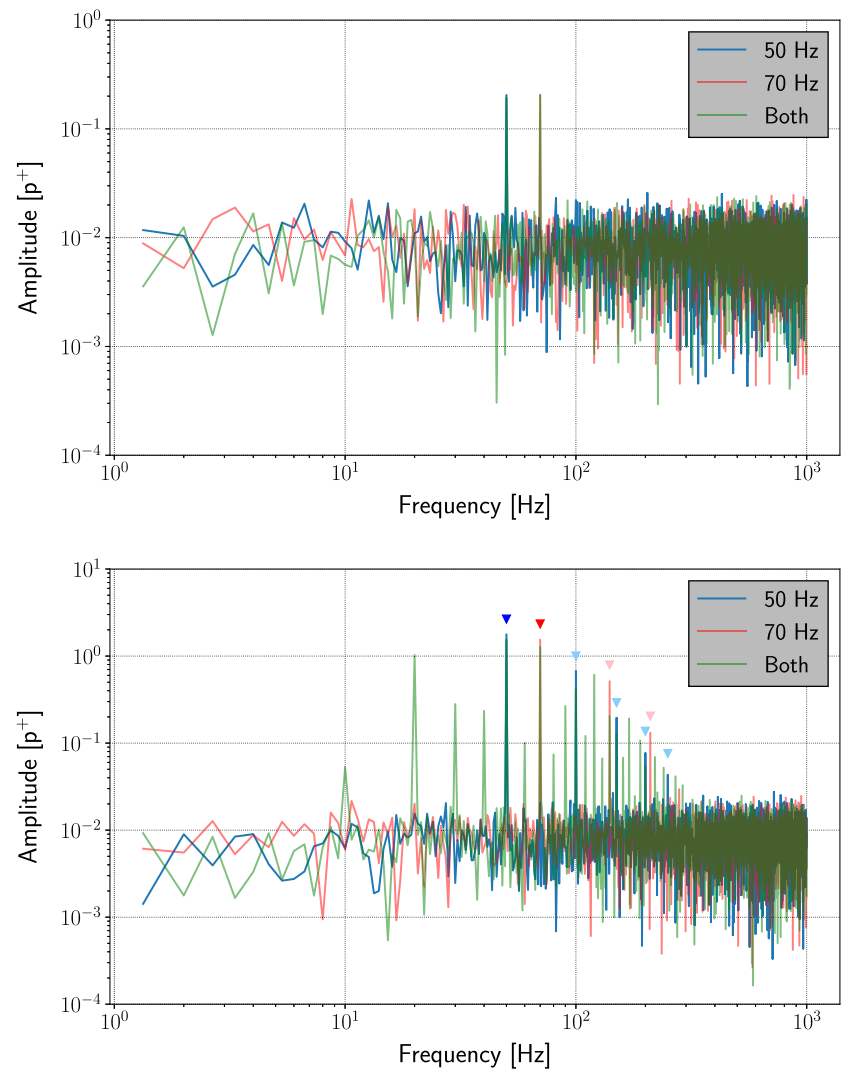

ramp are not enough to generate issues. For this reason, in this work, time invariance will be assumed valid for the frequencies of interest.

The available slow extraction MADX model can be exploited to gain a better insight on the problem of linear and nonlinear transfer function. Injecting two ripples at 50 and $70 \mathrm{~Hz}$ with equal amplitude, first below and then above the amplitude threshold of Eq. (6), the different behavior can be observed: the results are summarized in Fig. 3.

The two top plots of Fig. 3 show the results for the case of ripple amplitudes below threshold. It can be noticed that the extracted spill is in good approximation a square pulse (plus a noise component), and that the superposition principle holds, being the spectral component the same both when injecting the ripples separately and together. The two bottom plots show instead the case of input amplitudes above threshold. It is evident that the spill is no more a square-pulse-like signal, but is split in several bursts. This is a sufficient condition for harmonics of the main injected frequency to appear in the spectrum of the spill. Observing the spectrum of the case with the combined ripples (green), it can be noticed that the superposition principle does not hold anymore.

By referring to the transfer functions reported in Fig. 2 it is possible to notice that both 50 and $70 \mathrm{~Hz}$ are located
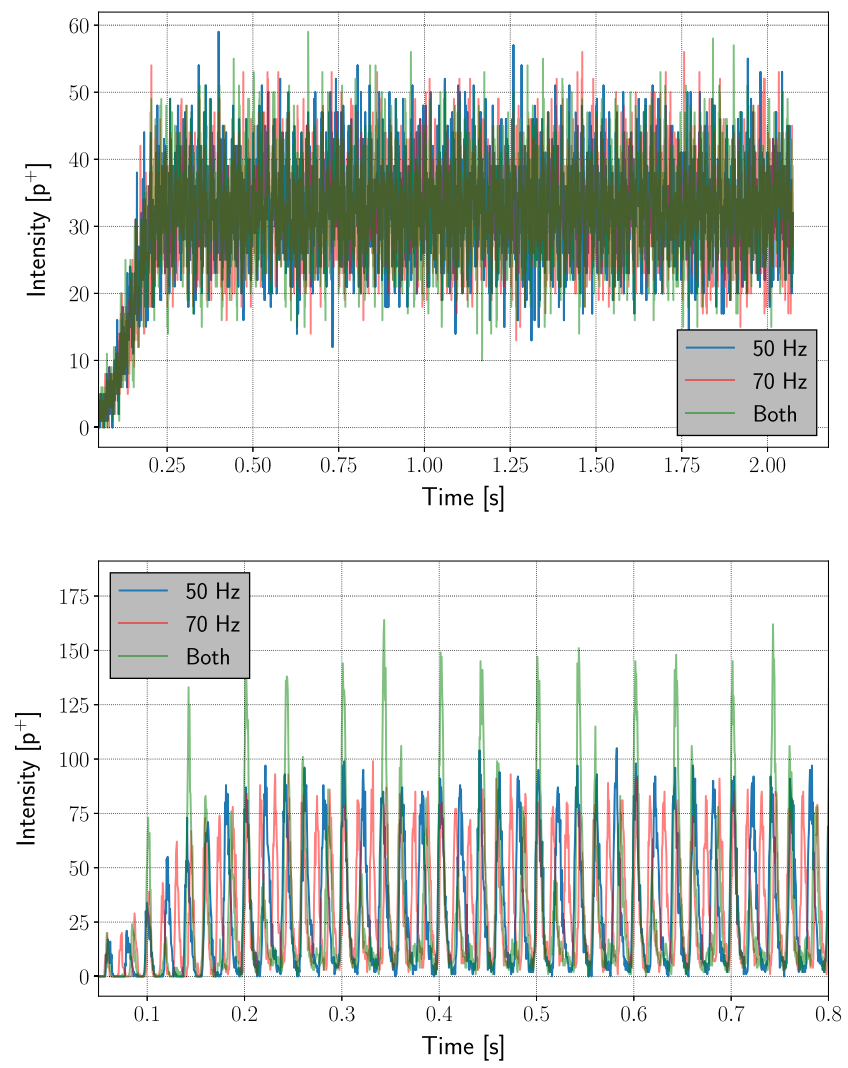

FIG. 3. Ripple signal spectra (left) and corresponding extracted spills (right) obtained by injecting 50 and $70 \mathrm{~Hz}$ ripple signals of equal amplitude, both separately and together. Top plots: ripple amplitudes below linearity threshold. Bottom plots: above linearity threshold; the single injected main frequencies and harmonics are highlighted respectively by full color and faded markers. 

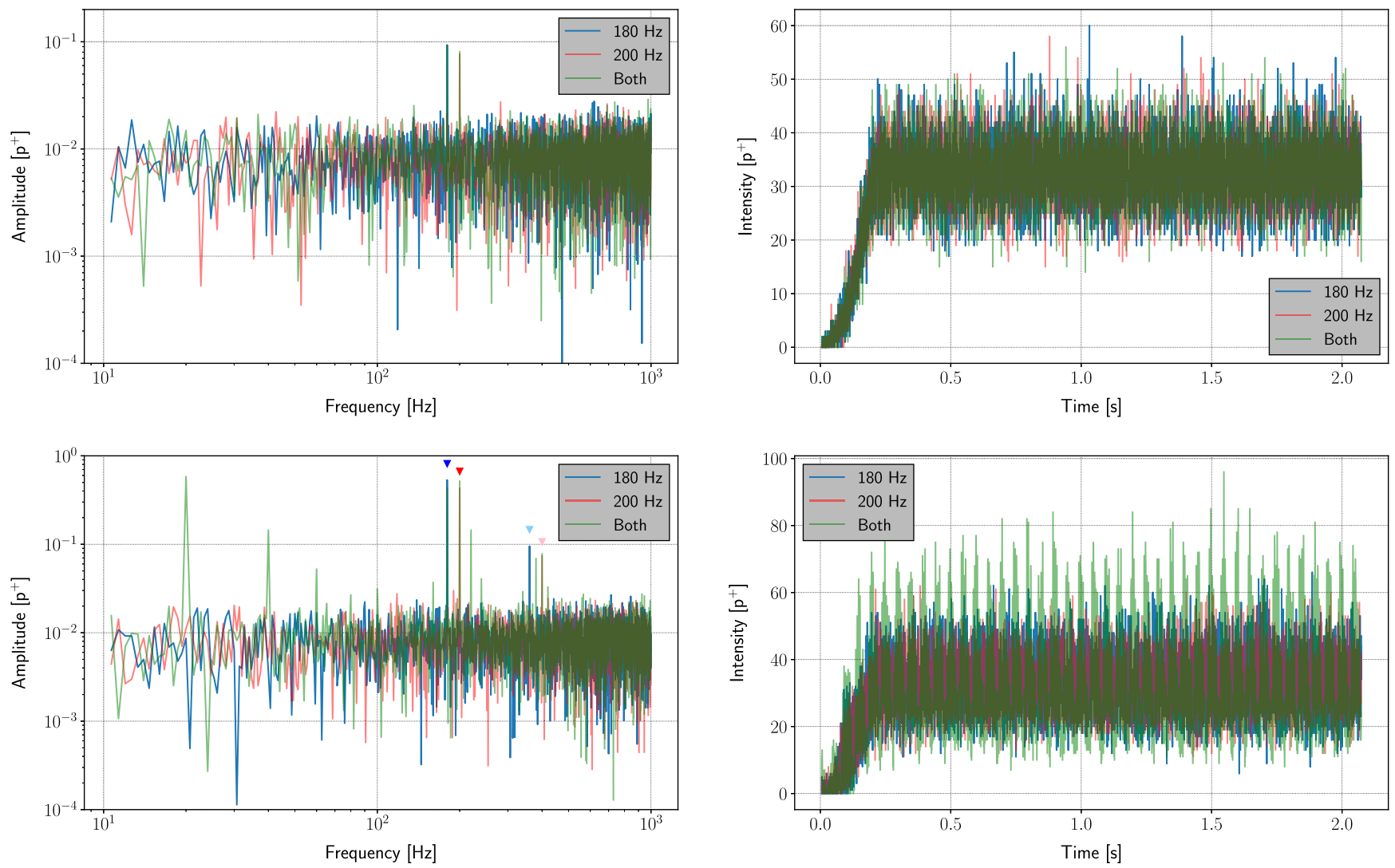

FIG. 4. Ripple signal spectra (left) and corresponding extracted spills (right) obtained by injecting 180 and $200 \mathrm{~Hz}$ ripple signals with equal amplitude, both separately and together. Top plots: ripple amplitudes below the linearity threshold. Bottom plots: above the linearity threshold; the single injected main frequencies and harmonics are highlighted respectively by full color and faded markers.

before the corner frequency of the low pass filter, meaning that the latter is not strongly acting on these frequencies. In order to investigate whether the low pass filter effect modifies the behavior observed in Fig. 3, the same superposition exercise is repeated for the frequencies of 180 and $200 \mathrm{~Hz}$, which are located well above the cutoff frequency. Figure 4 summarizes the results.

It is possible to notice from Fig. 4 that the trend is similar to the previous case of injection of 50 and $70 \mathrm{~Hz}$ : the superposition principle holds below the amplitude linearity threshold, while it is broken otherwise. However, a very important difference can be observed for the nonlinear input amplitudes: the effect of the low pass filter prevents the spill (bottom-right plot) from splitting into bursts, despite the injected input amplitude being the same as the previous case. Moreover, the bottom-left plot shows that only the first harmonic of the main frequencies is generated, as opposed to the case of Fig. 3. This comparison demonstrates how the low pass filter effect of the slow extraction process can be beneficial for the quality of the extracted spill: if properly exploited, it can be a very powerful tool to suppress the power supply ripples.

These results show that the transfer function is amplitude independent (and so implying a linear system, with all the benefits coming from it) only for small enough ripples satisfying the monotonicity of the tune ramp. In the opposite case, the system becomes nonlinear, and a unique transfer function for the process cannot exist anymore (in the proper sense of the term). For the nonlinear case, an operative procedure in the style of Fig. 2 can be developed to construct an empirical amplitude-dependent transfer function, for example, by using only the attenuation of the main frequency component of single-injected ripples. The procedure could be expanded to the harmonics of the main frequency, for an increased precision, but keeping in mind the violation of the superposition principle observed in Figs. 3 and 4.

As a natural continuation of the previous results, it is interesting to observe, from a transfer function formalism point of view, the spill quality improving technique implemented at GSI SIS-18 [31]. As already described in Sec. I, by injecting a dominant frequency ripple above the low pass filter cutoff frequency, it is possible to reduce the amplitudes of the lower frequency components (below the cutoff frequency, and hence more disruptive). The violation of the superposition principle, which has been shown in Figs. 3 and 4, is a necessary condition for this effect to take place. A simple example can be built 


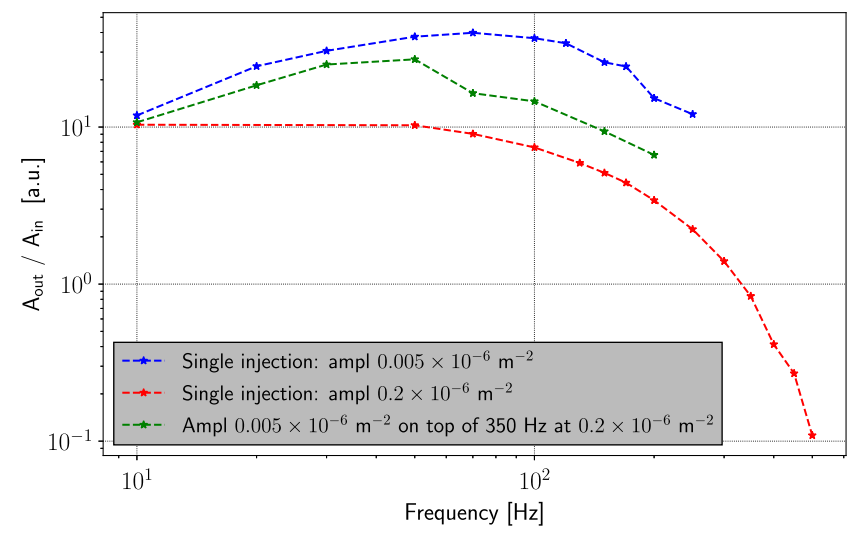

FIG. 5. The blue and red curves show respectively the linear transfer function and the $0.2 \times 10^{-6} \mathrm{~m}^{-2}$ fixed amplitude one, referring to Fig. 2. The green curve shows the transfer function for linear ripples injected together with a $350 \mathrm{~Hz}$ modulation at a nonlinear amplitude of $0.2 \times 10^{-6} \mathrm{~m}^{-2}$.

for the case of the SPS by injecting a sinusoidal ripple of $350 \mathrm{~Hz}$ with amplitude well above the nonlinearity threshold, together with other smaller frequency ripples in the linear regime. Intuitively, the high amplitude $350 \mathrm{~Hz}$ ripple will dominate the tune modulation, cutting away part of the smaller ones; since for the case of the SPS $350 \mathrm{~Hz}$ is above the low-pass filter cutoff frequency, the effect on the extracted spill of such ripple will be strongly attenuated. The resulting attenuation of the low-frequency linear ripples can be observed in Fig. 5.

The new transfer function of the superimposed ripples falls in between the two of the correspondent single injected signals (blue and red, respectively for linear amplitudes and nonlinear $350 \mathrm{~Hz}$ wave). The strongest attenuation with respect to the linear transfer function seems to be reached between 70 and $200 \mathrm{~Hz}$, where the ratio of the two reaches values of about 0.4. Going beyond this simple example, the picture gets more complicated and interesting, being the attenuation dependent on multiple parameters, as the position and amplitude of the dominant nonlinear ripple, and the frequency distance of the attenuated modulations from the dominant ripple. On top of this, the amplitudes of the attenuated ripples can become a parameter too, especially if they start to cross the nonlinearity threshold. This could be treated as an optimization problem in order to find the best combination of parameters for the maximum attenuation of the desired frequencies.

The advantage of using such a technique to improve the spill quality comes especially when the experiments using the extracted beam are not sensible to frequency components above a certain threshold, and so a new dominant frequency can be introduced without consequences (synchrotron modulation and bunched extraction can attenuate the ripples in the same way too). Unfortunately, this is not the case for the SPS, in which every strong frequency component disturbs the experiment's detectors. For this reason, further studies are needed to evaluate the actual benefit of this technique for the SPS case, and it will not be covered hereafter.

To further investigate the origin of the nonlinearity, the input for the transfer function model could be changed from the quadrupolar current to the ideal spill of Eq. (4). As the latter does not include the low-pass filter effect, a restored linearity due to such input change would imply that the process can be modeled by two cascaded effects: a peak detector derivator [Eq. (4)] and a linear low-pass filter. For a nonlinear ripple some complication is introduced, as the input spectrum would not consist anymore in a single frequency component, but would count a number of harmonics of the main frequency. In this case, a transfer function can be computed by only using the main Fourier component of the input signal, knowing that in case of restored linearity all the other transfer functions computed using its harmonics should give the same result. The results relative to the same input ripples used for Fig. 2 are reported in Fig. 6.

While the small amplitude transfer functions are brought nearer to each other with respect to the case of Fig. 2, a nonlinear behavior is still evident, being the obtained transfer functions still amplitude dependent. These results show that the nonlinearity is not only coming from Eq. (4), but either it is also present in the low-pass filter effect of the slow extraction, or the two effects cannot be disentangled in such a way. The attempted change of input signal does not seem enough to restore linearity, and since it carries with it some complications with respect to the differential approach stated in Eqs. (1) and (2) (as having to process the input ripples together with the tune ramp), it will not be used any further. Additionally, it can be also shown that this change of input is not enough to explain the attenuation observed in Fig. 5.

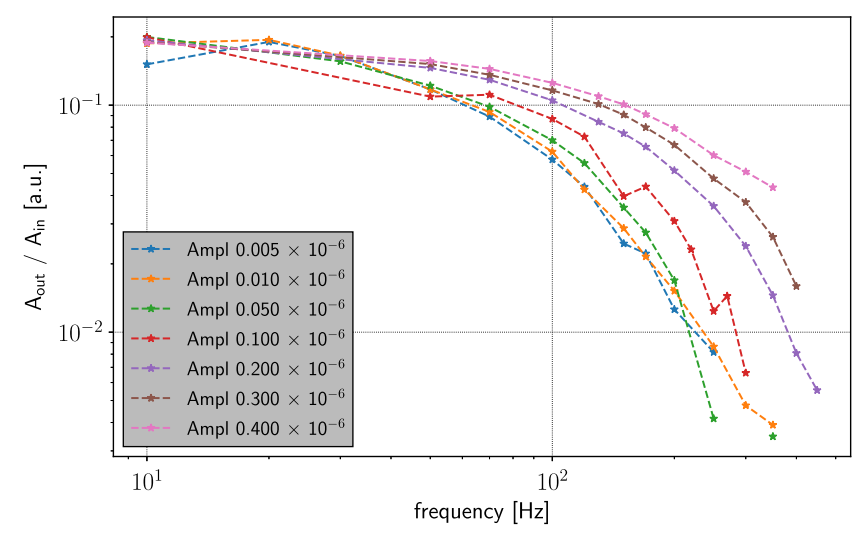

FIG. 6. Fixed amplitude transfer functions from the rectified derivative of the focusing quadrupole strength to the extracted spill. The injected sinusoidal amplitudes are reported in the legend in units of $\mathrm{m}^{-2}$. 


\section{Magnet strength to tune conversion}

The second gray block of Fig. 1 represents the transfer function from focusing quadrupole to horizontal tune. It is possible to verify from simulation that a sinusoidal ripple on the main focusing quadrupoles leads to a samefrequency sinusoidal ripple on the tune ramp with good approximation, the only deviation coming from a small residual dependence of the tune on the quadrupole ramps. Given this, a proportionality law between the focusing quadrupole ripple and the correspondent tune one will be assumed (the additional strength dependence could easily be added in case of required increased precision). The proportionality constant has been computed using the MADX model of the SPS, and also cross-checked from the calibration tables used for the machine operation.

\section{Tune to spill simulation results}

In order to isolate the results of the tune-to-spill transfer function block (referred to Fig. 1) a 2D Henon map [38] model has been developed. The model can be expressed as follows:

$$
\left(\begin{array}{c}
X \\
X^{\prime}
\end{array}\right)_{n+1}=\mathbf{R}\left(2 \pi Q_{n}\right)\left(\begin{array}{c}
X \\
X^{\prime}+V_{\mathrm{ss}} X^{2}
\end{array}\right)_{n},
$$

where $\left(X, X^{\prime}\right)_{n}^{T}$ are the normalized (Floquet, emittancepreserving) horizontal phase space coordinates at the $n$th turn, $V_{\mathrm{ss}}$ is the normalized virtual sextupole strength for the SPS, $\mathbf{R}(\theta)$ is a rotation matrix of angle $\theta$, which in Eq. (7) coincides with the linear one-turn map, and, finally, $Q_{n}$ represents the tune value at the $n$th turn. All the internal parameters of this model (e.g., phase space and momentum distribution, tune, chromaticity, septum coordinates) are matched to the correspondent ones from the full MADX simulation. Similarly, a linear tune ramp is used to extract particles along their fixed momentum distribution, for the same flattop duration. In order to inject amplitudes of tune ripples corresponding to the focusing quadrupole strength amplitudes injected in the MADX model, the conversion constant discussed above is used.

Figure 7 shows the simulation results. These results are very similar to what has been obtained using the full SPS MADX model described in Sec. II A 1 and reported in Fig. 2. In particular, the same considerations apply also for this case.

Figure 8 shows a direct comparison of the fixed amplitude transfer functions obtained from the MADX extraction model and the Henon-based model. It can be noticed that the main difference comes from a not perfect matching of the ripple amplitudes between the two models. However, the small-amplitudes linear transfer functions of the two models can be rescaled to be fully compatible as shown in the small plot inside Fig. 8. Being the smallamplitudes transfer function univocally defined and

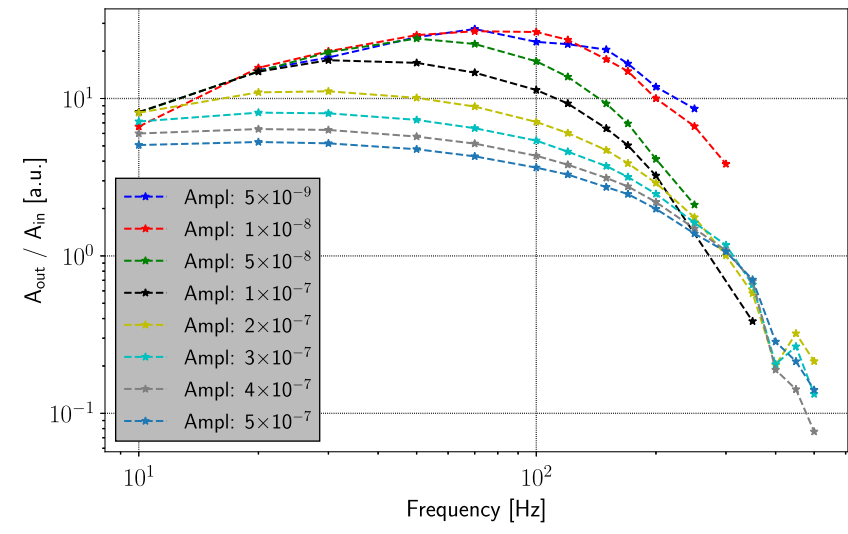

FIG. 7. Transfer functions for different sinusoidal ripple amplitudes obtained from the tune-to-spill Henon map-based simulation.

independent of the input, this result shows that the Henon map model successfully captures the essence of the frequency transfer process of slow extraction.

\section{B. Effective transfer map}

Using the results obtained up to now, an empirical semianalytical model can be built in order to predict the frequency response of the slow extraction from a given current signal. The transfer functions extracted from the MADX model have been fitted using different low pass filter functions of the type

$$
\begin{aligned}
& F(s)=\frac{K \cdot f(s)}{\Pi_{i}\left(1+a_{i} s+b_{i} s^{2}\right)} \\
& f(s)= \begin{cases}s & \text { linear ripples } \\
1 & \text { nonlinear ripples }\end{cases}
\end{aligned}
$$

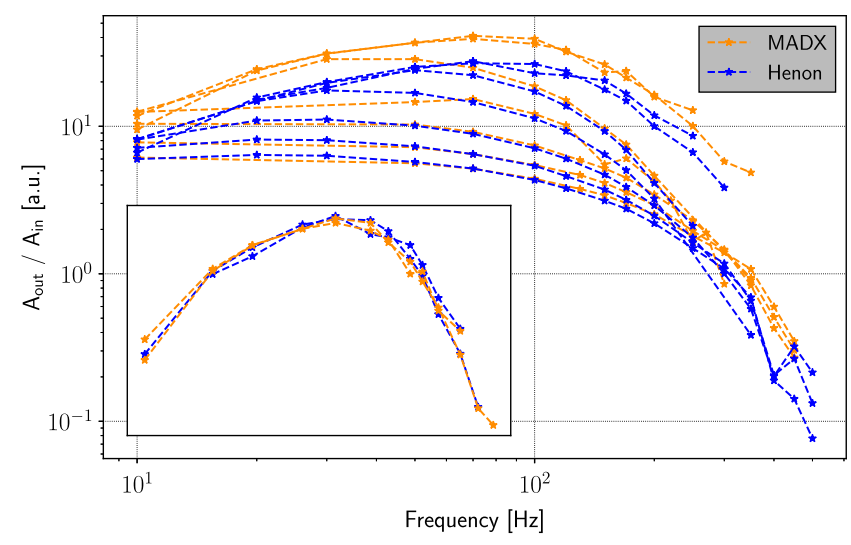

FIG. 8. Fixed ripple amplitude transfer functions obtained for the Henon map simulation and for the full strength-to-spill MADX model (respectively reported in Figs. 7 and 2). The small plot shows the full compatibility between the Henon and MADX smallamplitudes transfer function after rescaling the Henon one by a constant. 


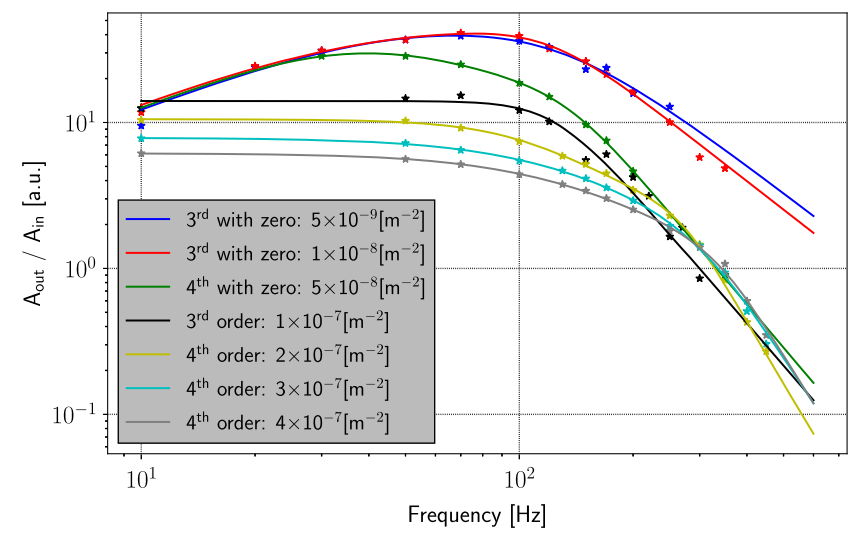

FIG. 9. Fixed amplitude transfer functions fitted with curves of the type of Eq. (8). The legend refers to the order of the low-pass filter which gives the best fit.

where $s \in \mathbb{C}, a_{i}, b_{i}$ and $K$ are real coefficients, $f(s)$ introduces a zero for the linear case, and the range of $i$ depends on the particular order of the low pass filter. Every obtained transfer function can be fitted with a function of the type of Eq. (8), as shown in Fig. 9.

The order of the low pass filter grows with the input amplitude, starting from a second order asymptotic behavior for the linear case, to a fourth order of the maximum simulated amplitude. For the nonlinear case, the transfer functions are only relative to the main frequency (i.e., there are also its harmonics, as shown in Figs. 3 and 4) and the spill can potentially be split in several pulses: the linear and nonlinear models shall not be compared directly since they can refer to two different conditions.

For the case of the linear small-amplitude transfer function, a good fit is given by the function

$$
F_{3 z}(\omega)=\frac{K \cdot \omega}{\sqrt{\left[1+\left(\frac{\omega}{\omega_{c}}\right)^{2}\right]\left\{\left[1-\left(\frac{\omega}{\omega_{c}}\right)^{2}\right]^{2}+4 \zeta^{2}\left(\frac{\omega}{\omega_{c}}\right)^{2}\right\}}}
$$

with the effective pole at $\omega_{c} / 2 \pi \simeq 90 \mathrm{~Hz}$ for the case of the SPS (eventually, two poles $\omega_{c 1}$ and $\omega_{c 2}$ can be defined for more flexibility).

In the nonlinear regime, the transfer function will depend also on the input amplitude, hence a two-dimensional map is needed to represent a complete transformation from current to spill. It can be found that the variation with amplitude for fixed-frequency transfer function points approximates a power law. In order to keep into account of the deviations from the law (as the behavior is less fitting than the fixed amplitude case), the full nonlinear transfer function is obtained using a double logarithmic linear interpolation of the fitted low pass filter functions of Fig. 9 along the amplitude dimension. Figure 10 shows the obtained normalized quadrupole strength to spill nonlinear transfer map.

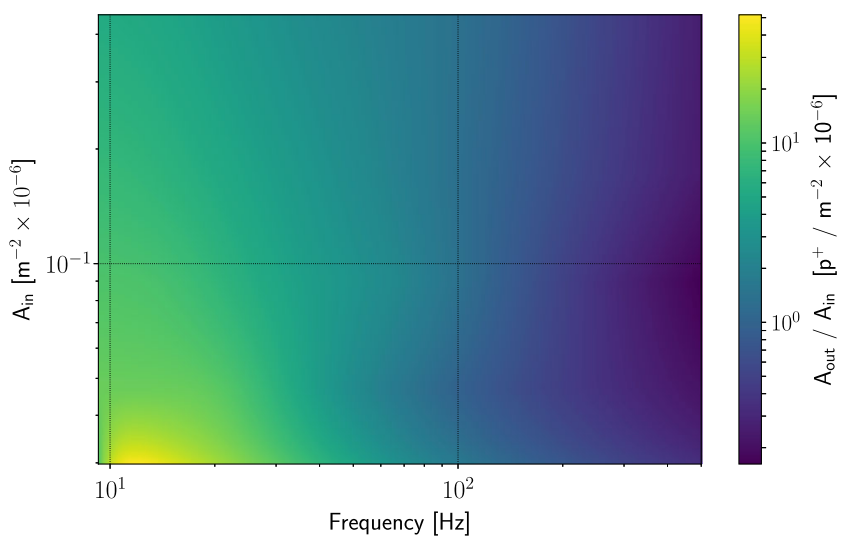

FIG. 10. Two-dimensional transfer map obtained from the fitted transfer functions of Fig. 9, and a bilogarithmic linear interpolation. The abscissas and ordinates are respectively the injected ripple frequency and amplitude (in units of normalized quadrupole strength), while the color code shows the transfer function value.

The same procedure could be repeated for the other harmonics of the main injected frequency on the extracted spill, obtaining a 2D transfer map for every harmonic.

The nonlinear transfer map model holds for singleinjected nonlinear ripples, while it should be revised in case of superposition of ripples, given the violation of the superposition principle shown in Figs. 3 and 4. In particular, while from the latter plots the main injected amplitudes seem to remain the same, this is not guaranteed in general (as shown by the superposition of linear and nonlinear ripples in Fig. 5). On top of this, also more frequency components, as the difference of the two injected frequencies with its harmonics, will appear.

\section{DEDICATED RIPPLE INJECTION MEASUREMENTS}

A dedicated data taking campaign was carried out at the SPS in order to validate the above-developed model. The measurements were taken by connecting a voltage waveform generator to the power converters of the main focusing quadrupoles, so to inject a sinusoidal perturbation at arbitrary frequency and amplitude. The circulating intensity in the machine was set to the value of $\sim 4 \times$ $10^{11}$ protons, that is about 2 orders of magnitude smaller than nominal, for machine protection reasons related to other parallel measurements. The recorded quantities for each extraction cycle are (i) amplitude and frequency of the injected voltage ripples from the waveform generator; (ii) measured input current of the focusing quadrupoles and its correspondent reference current, both sampled at $1 \mathrm{kHz}$; and (iii) time profile of the extracted proton spill, measured with a secondary emission monitor placed just after the extraction septa (the beam intensity signal is sampled at $2 \mathrm{kHz}$ ). 


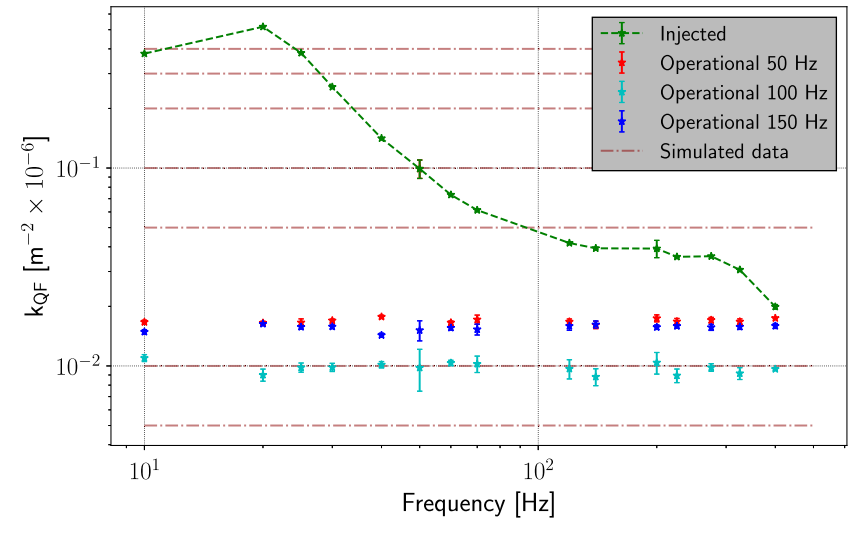

FIG. 11. The green points (connected by dashed lines) show the strength-equivalent ripple amplitude and frequency of the sinusoidal ripple injected using the waveform generator. The red, light blue and blue points show the 50, 100 and $150 \mathrm{~Hz}$ harmonics components on the measured current coming from the power supply ripples. The horizontal lines are the ripple amplitudes simulated in Sec. II A. All the points that generated the green curve have been injected as voltage ripples of fixed $240 \mathrm{mV}$ amplitude.

The noise on the data, $\Delta i$ [Eq. (1)], is calculated by subtracting the reference current from the measured one, and performing a spectral analysis. In order to compare the experimental data with simulations, it is necessary to convert the experimentally injected ripple amplitudes from units of current to quadrupole strength: this has been done using available calibration tables (see for example [39]). An overview of the measurements is shown in Fig. 11.

In particular, all the injected ripples fall into the nonlinear regime, and therefore this data can be used to validate both the full MADX simulation model and the empirical 2D transfer map developed above. All the injected data shown in Fig. 11 has been used as input for the simulations, both using the MADX model and the empirical 2D transfer function of Fig. 10.

Figure 12 shows the comparison between the transfer function points obtained from experimental data, MADX simulations, and 2D empirical transfer map (all from single frequency analysis). It is possible to notice that for all but two frequency values $(120$ and $140 \mathrm{~Hz})$ the experimental data points are in a very good agreement with both the models. Moreover, the MADX simulation results are almost perfectly agreeing with the empirical map in all but two points, where anyway the fractional difference is smaller than $10 \%$. These results show the robustness of the developed empirical map, especially considering that the map has been built using only five fixed-amplitude transfer functions (the nonlinear ones of Fig. 2). By simulating more fixed-amplitude transfer functions, the accuracy of the empirical transfer map can be improved up to a desired level. As no systematically increasing reduction of the experimental transfer function is evident from Fig. 12, no

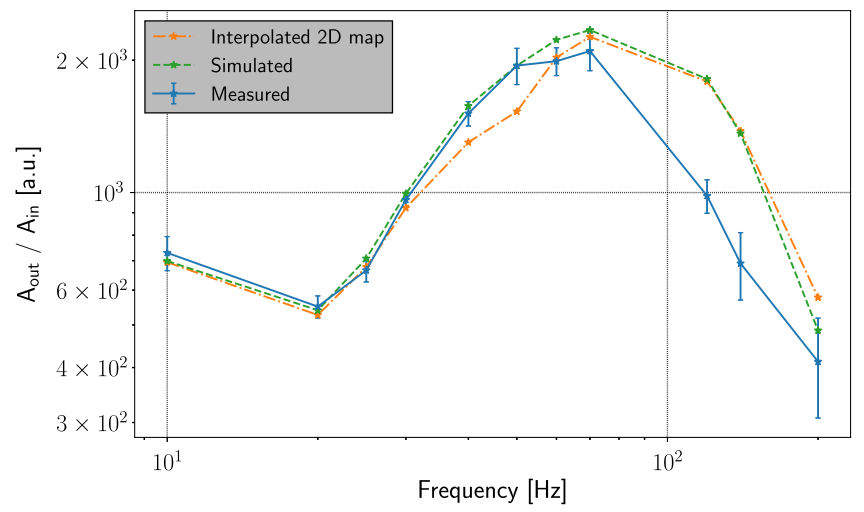

FIG. 12. Comparison between the transfer function points from the experimental data (blue continuous line), the full MADX simulation model (dashed green line), and the 2D empirical transfer map (dot-dashed orange line).

hardware effect (i.e., vacuum chamber shielding and magnet losses, referred to Fig. 1) can be said to take place in the observed frequency region. This would be in agreement with hardware estimations, which would place these effects at the $\mathrm{kHz}$ level.

The reason why less injected frequency points are shown in Fig. 12 with respect to Fig. 11 is that, for injected frequencies above $200 \mathrm{~Hz}$, different methods used to process the spill spectra lead to systematically different results; this fact is most probably due to the very low extracted intensity, which makes problematic the analysis of small and suppressed frequency components. Finally, the reason of the low compatibility between data and model for the points at 120 and $140 \mathrm{~Hz}$ is not clear: given the good agreement for the rest of the points, it could be related to some unnoticed problem during the data taking.

\section{OPERATIONAL DATA}

As for the dedicated measurement campaign described in Sec. III, also for operational data the measured current, reference current, and spill intensity profile are recorded for every extraction cycle. The extracted intensity is about $3 \times$ $10^{13}$ protons per spill. For these reasons, operational data makes for another useful opportunity to validate the developed simulation models.

Figure 13 shows the average spectrum of the focusing quadrupole ripple current (converted in units of quadrupole strength) and the corresponding measured spills during a stable physics run of two days. As introduced in Sec. I, the $50 \mathrm{~Hz}$ and its harmonics are the frequency components impacting the spill the most during operation. Their typical amplitudes are below $2 \times 10^{-8} \mathrm{~m}^{-2}$ (the averaged values of the 50, 100 and $150 \mathrm{~Hz}$ peaks are also shown in Fig. 11). These amplitudes are significantly smaller than those injected during the dedicated measurements, and, according to the simple case of Eq. (6), they should be below the linearity threshold of the transfer function. 

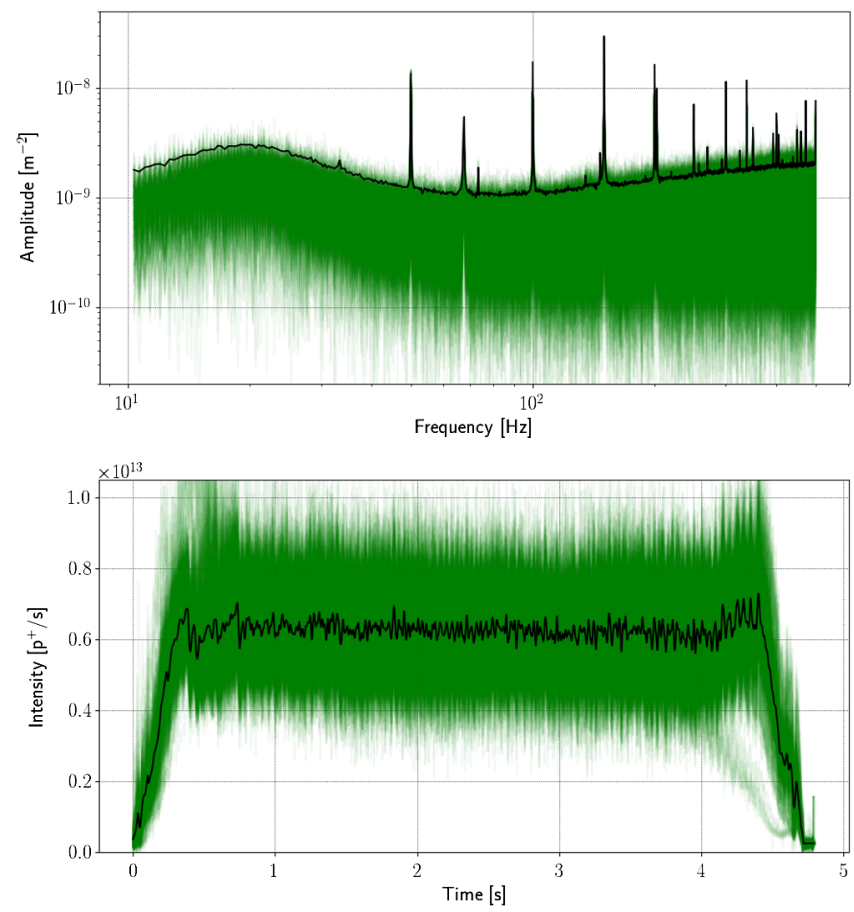

FIG. 13. Single measurements (green lines) and their average (black line) for the ripple quadrupole current (top plot) and extracted spill (bottom plot) during a physics run.

Unfortunately, under these conditions, it can be shown that a non-negligible component of measurement noise is present on the measured current ripples. This makes it challenging to precisely estimate the actual frequency components on the magnets. In particular, the lack of information on the relative phase between the measurement chain discrete spectral components (e.g., $50 \mathrm{~Hz}$ and harmonics) and the actual ones present on the magnets does not allow a precise estimation of the latter. For this reason, only the continuous-noise transfer function can be computed from the operational data, as opposed to the single frequencies discrete transfer function computed up to now. Under linearity conditions, the two methodologies should lead to comparable results.

To correctly compute the experimental continuous transfer function, the measurement noise needs to be removed from the logged current, in order to have an input spectrum as realistic as possible. There are different ways to attempt this, and the best one has been found in using the theoretical frequency response between measurement noise and actual magnet current, based on a model of the magnet at small-tomedium size voltages which was reproduced quite closely by measurements at the end of 2017 [40]. The frequency range of the model is limited to about $167 \mathrm{~Hz}$, i.e., the Nyquist frequency of the control system. By applying this frequency response function to the measurement noise spectrum (which has been measured), and keeping into account the presence of a white noise floor for large enough

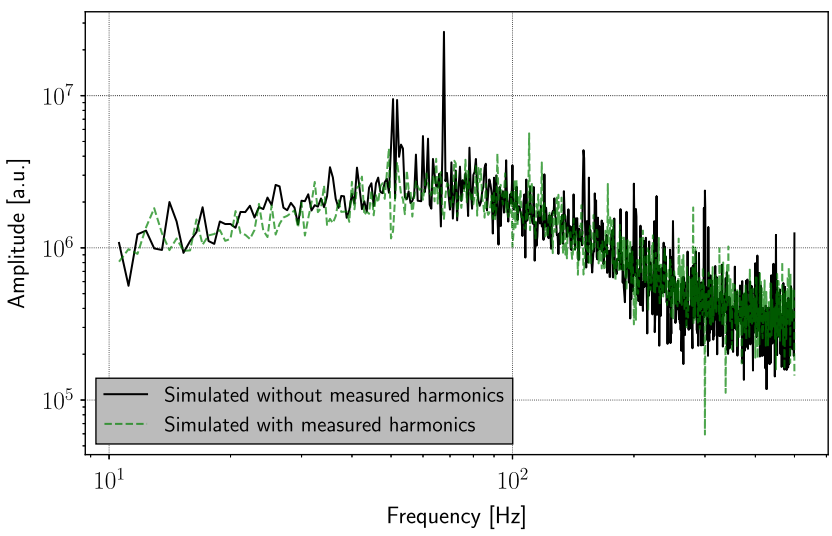

FIG. 14. Comparison between simulated operational transfer function obtained by including the harmonic peaks in the simulation (dashed green line) or not (black line).

frequencies, the expected current in the magnets could be obtained.

In order to compare the operational data with the model, a simulated version of the operational continuous transfer function is obtained by using the measured current as an input for the MADX simulation. It has been previously observed that in case of ripples with amplitudes above the linearity threshold, the obtained transfer function is strongly dependent on the input signal. For the operational input current (e.g., Fig. 13) the only components of the spectrum that could potentially break the linearity are the main harmonics. The harmonics present on the magnets should have the same order of magnitude of the ones seen in the measured current: this implies that the linearity condition should hold. Under these conditions, the simulated transfer function should not be strongly dependent on the input, meaning that, even if the simulated current is dominated by measurement noise, the correct result should be obtained. The latter hypothesis is tested by simulating the measured current with and without the main harmonics, and comparing the two resulting transfer functions. Figure 14 shows that no significant difference can be observed, confirming the hypothesis of linearity.

The good agreement between the experimental and simulated continuous transfer functions can be observed in Fig. 15. The harmonic peaks visible in the measured transfer function are an artifact of the analysis process: only the continuous spectrum has been used. Despite the satisfactory result, further investigations on the measurement noise and its suppression will be required both to validate the outcome of Fig. 15 and to correctly estimate the discrete frequency components present on the magnets.

Other systematic effects, which could have an influence on the final result, will also have to be investigated in the future, as the servo-spill system used to suppress the main $50 \mathrm{~Hz}$ components (this system is always active for operational data, while it has been turned off for the dedicated measurements of Sec. III), or the presence of 


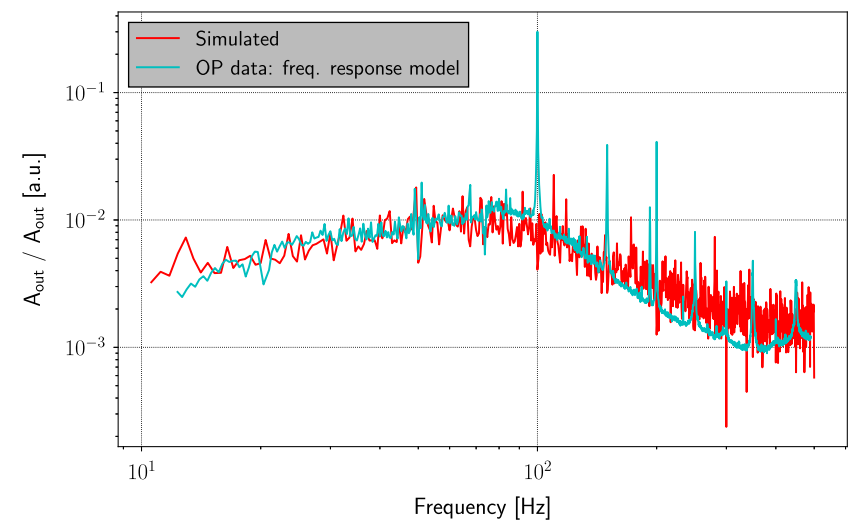

FIG. 15. Measured operational spill transfer function (light blue) compared with the simulated one (red).

strong ripples with frequencies higher than $500 \mathrm{~Hz}$, being $500 \mathrm{~Hz}$ the Nyquist frequency of the current measurement system.

\section{MODEL APPLICATION}

The simulation model developed and validated in the previous sections has been built using the nominal optics and extraction parameters of the SPS. In this section, the developed simulation model will be used to explore different machine parameters, looking for the possibility to improve the spill microstructure of the slow extraction.

The parameters that can be varied and affect the spill quality are the machine chromaticity $\xi$ and the extraction sextupole strength $V_{\mathrm{ss}}$. Increasing the virtual sextupole strength increases the particle speed and acceleration in phase space, and reduces the spread of the interval distribution (Chapter 3 of [41]). Decreasing it will have the opposite effect, hence increasing the attenuation of high frequencies, as also shown in [22,23]. Regarding the chromaticity, its effect is double. Let us consider a sinusoidal ripple $r(t)$ superimposed to an ideal tune ramp, with a uniform momentum distribution of the particles. Under the assumptions of a monotonic tune and instantaneous extraction, the spill can be expressed as

$$
\frac{d N}{d t}=K \frac{d Q}{d t}=K \frac{\Delta Q}{\Delta T}+K \frac{d r}{d t},
$$

where $K$ is the particle density in the tune space. In a typical situation in which the slow extraction has a fixed length and a fixed number of particles, the total tune variation $\Delta Q$ will be a function of the chromaticity as

$$
\Delta Q=|\xi| \Delta \delta_{p}
$$

hence, if $A_{r}$ is the sinusoidal ripple amplitude, the ratio between the rippled and flat components [from Eq. (10)] of the spill becomes

$$
\frac{K 2 \pi f A_{r}}{K \Delta Q / \Delta T}=\frac{2 \pi f A_{r} \Delta T}{|\xi| \Delta \delta_{p}} \propto \frac{1}{|\xi|}
$$

meaning that the higher the chromaticity the more attenuated the ripple on the spill gets.

By rescaling the tune variation according to Eq. (11) (i.e., in order to maintain a fixed extraction duration and number of extracted particles), the slope of the tune ramp $\Delta Q / \Delta T$ increases proportionally to the chromaticity. This implies that by increasing the chromaticity the extraction process becomes intrinsically more resilient to ripples, given that the faster the tune ramp the larger the ripple amplitude needed to significantly alter the spill. In particular, the ripple nonlinearity (or full modulation) threshold increases [Eq. (6)].

The Henon map model developed in Sec. II A 3 is used for performing the parametric scan. This simulation model is significantly faster than MADX: on average, there is more than a factor 100 between their simulation times. The range of the scan parameters is delimited by realistic reduction and increase factors of 2 with respect to the nominal values $\xi_{0}(\simeq-26.67)$ and $V_{\mathrm{sS}}^{0}\left(\simeq 169.3 \mathrm{~m}^{-1 / 2}\right)$. The scan points are taken over a $10 \times 10$ uniform grid over the chosen parameter space. For every $\left(V_{\mathrm{ss}}, \xi\right)$ pair, a full transfer function of 13 frequency points (from 10 to $500 \mathrm{~Hz}$ ) is simulated using the Henon map model. The simulated ripple amplitude has been chosen small enough to fulfill the linearity condition, so to allow to neglect the dependence on amplitude of the transfer function. The detail of all the generated transfer functions is shown in Fig. 16, where it can be observed that about 1 order of magnitude is spanned along the $Y$ axis, while the position of the pole spans a few tens of Hertz. This shows that significant improvements in ripple suppression can potentially be achieved.

For a characterization of the best sextupole strengthchromaticity combinations, 2D maps of the suppression of each of the main harmonics are reported in Fig. 17. Attenuations of the $50 \mathrm{~Hz}$ component of a few folds seem achievable, up to a maximum suppression of about a factor 3. The attenuation increases for higher harmonics.

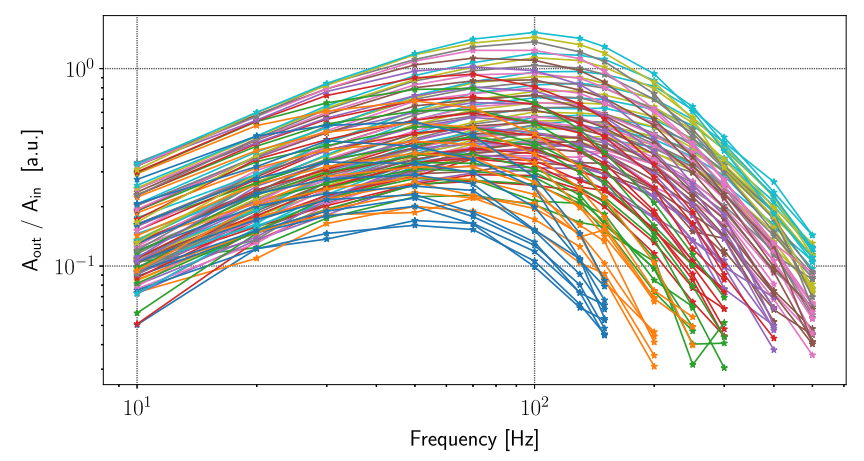

FIG. 16. Overview of all the obtained transfer functions from the $V_{\mathrm{ss}} \times \xi$ parameter scan, in the range from $1 / 2$ to 2 times the nominal values. 

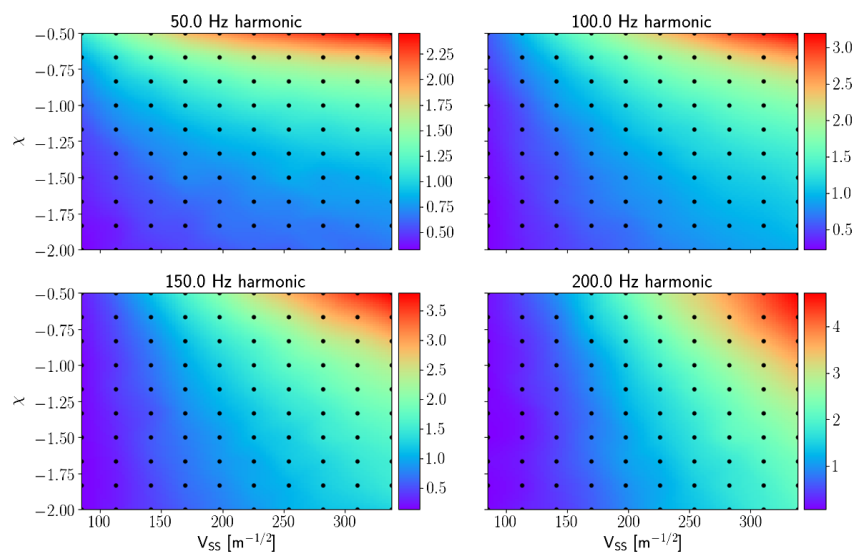

$200.0 \mathrm{~Hz}$ harmonic

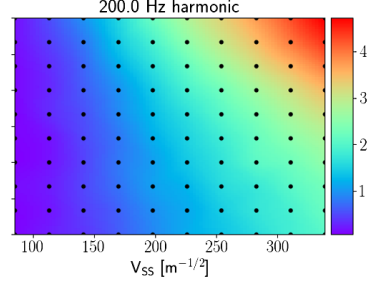

FIG. 17. Interpolated 2D maps of the main harmonics suppression factors in the $V_{\mathrm{ss}} \times \xi$ scan. The variable $\chi$ (normalized chromaticity) is defined as $\xi / Q_{0}$, where $Q_{0}$ is the nominal machine tune. The color code indicates the ratio between the obtained transfer function value and the nominal one for the considered harmonic component. The black points show the scan grid.

The results of Fig. 17 confirm what was expected: higher chromaticity (in absolute value) and weaker sextupole strength increase the attenuation of the slow extraction transfer function.
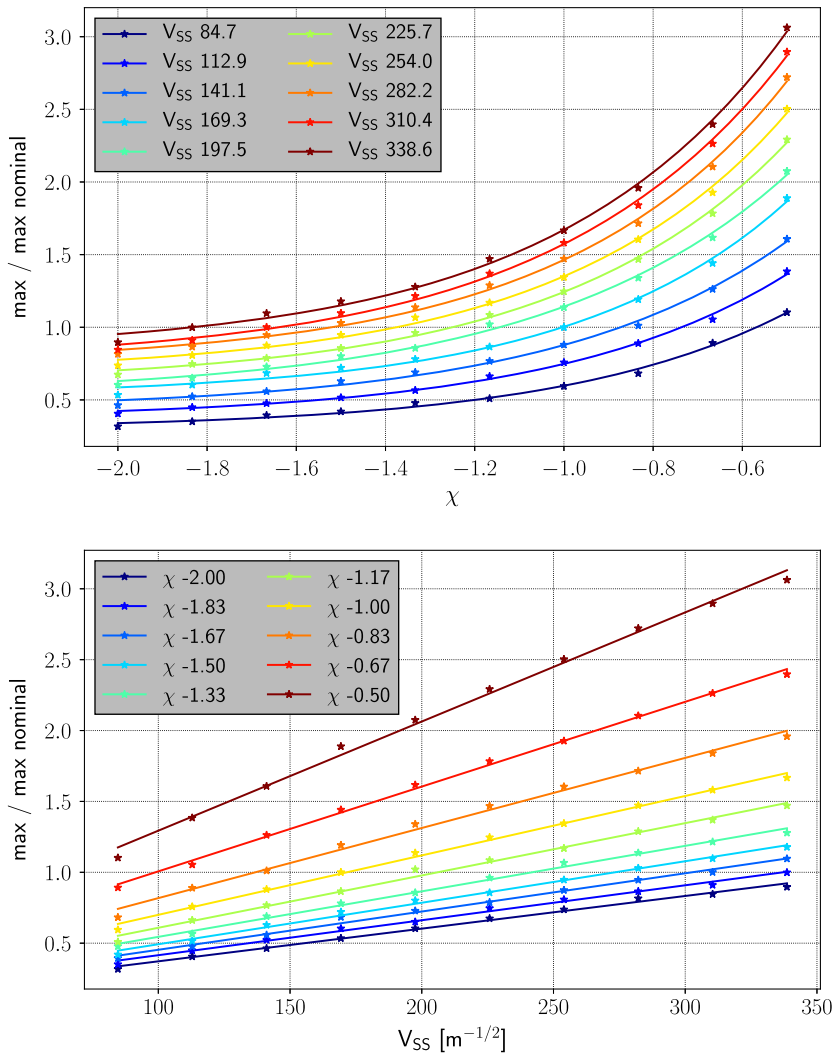

FIG. 18. 1D projections of the transfer function maximum, normalized to the one of the nominal SPS working point. Top: fixed $V_{\text {ss }}$ curves as a function of $\chi$. Bottom: fixed $\chi$ curves as a function of $V_{\text {ss }}$.
A direct parametrization of the transfer function can be performed by observing the position of the pole and the value of the maximum as a function of the chromaticity and sextupole strength. Both of these parameters strongly determine how good is the obtained transfer function in terms of ripple suppression.

Figure 18 shows the one-dimensional projections of the normalized transfer function maximum. The continuous lines are the results of a fit of each curve. In particular, for the fixed $V_{\mathrm{ss}}$ curves an exponential law (plus constant) yields a good fit in the range of interest. This is not far from the hyperbolic behavior foreseen by the simple example of Eq. (12). For the fixed chromaticity curves, a linear law is found as the best fit. Starting from the fit results shown in Fig. 18 it would be straightforward to develop an effective analytic model which could be used for fast estimations, for instance by modeling the dependencies of the fit parameters in $V_{\mathrm{ss}}$ and $\xi$. In fact, it can be shown that the latter dependencies can be approximated by analytic functions.

The same procedure can be applied for the transfer function pole, of which the 1D projections are shown in Fig. 19.

The result is not as smooth as the one of Fig. 18; this is only because estimating the correct position of the pole is a
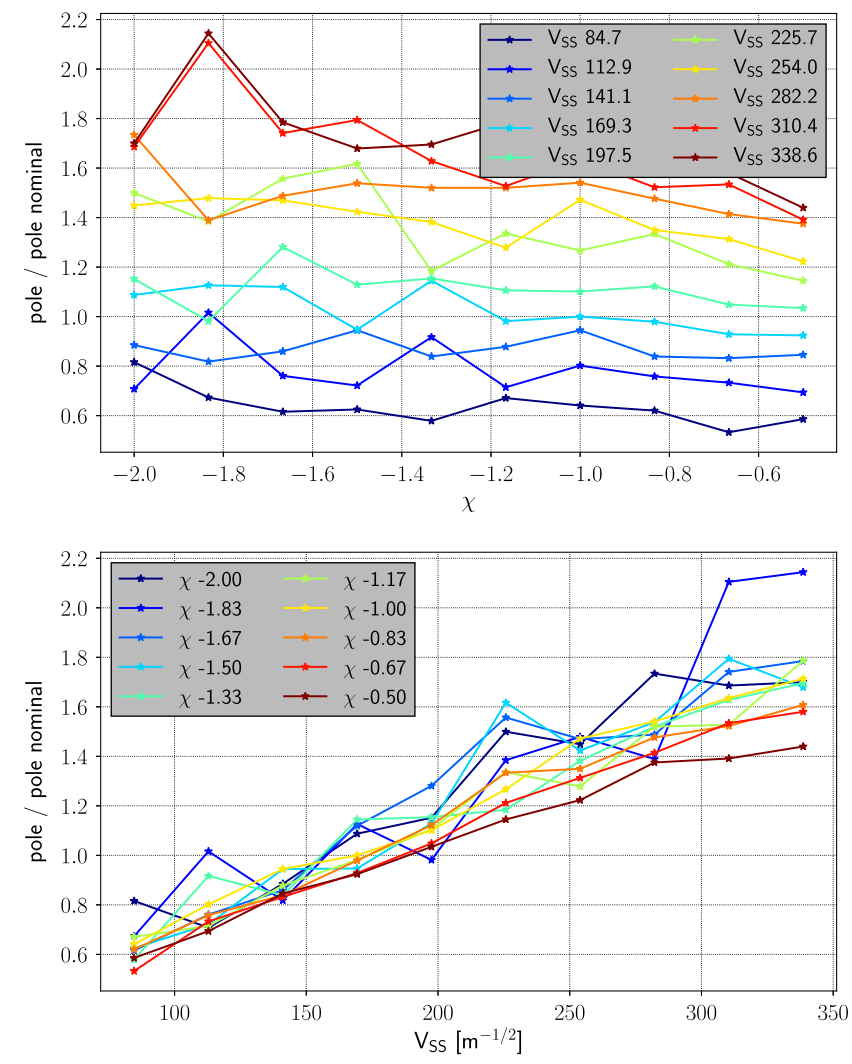

FIG. 19. 1D projections of the transfer function pole, normalized to the one of the nominal SPS working point. Top: fixed $V_{\mathrm{ss}}$ curves as a function of $\chi$. Bottom: fixed $\chi$ curves as a function of $V_{\mathrm{ss}}$. 
more error prone process, especially when automatized to many transfer functions which can significantly variate in shape (if needed, it could be solved by implementing more sophisticated analysis algorithms). However, it is evident that the main dependence is on the $V_{\mathrm{ss}}$ parameter. A reduction of the position of the pole of about a factor 0.7 seems within reach. For reference, the nominal pole is located at about $90 \mathrm{~Hz}$. Both 1D projections can be fitted using a linear model. Once again, an effective analytical model of the transfer function pole as a function of the scan parameters could readily be extrapolated from the fits of the $1 \mathrm{D}$ projections. The result is a linear behavior on the virtual sextupole strength, with a small linear dependence on the chromaticity which could be neglected in first approximation. This result follows the trend observed in the simple model developed in Chapter 3 of [41].

\section{EXTRAPOLATION TO OTHER MACHINES}

All the methods presented up to now could be applied in a straightforward way to any other accelerator, so to characterize its frequency response. In particular, the Henon map-based model used for the extraction parameters scan can be readily used without the need of full lattice files or writing new code in dedicated particle-tracking programs (e.g., MADX): it just requires the global and slow extraction specific machine parameters. This allows to simulate different machines as easily as performing the parametric scan of Sec. V. By doing so, even if dedicated validations would be required for each specific case, it follows that significant differences in the transfer function characteristics of different machines are to be expected. For instance, a significant influence is given by the revolution time (which sets the time units and so the overall position of the pole), the employed extraction method, and the particular values of the extraction parameters. On top of this, a different response of the hardware components would need to be taken into account.

\section{CONCLUSION}

In the present paper the frequency transfer properties of the slow extraction for the SPS have been investigated. A SPS slow extraction MADX model has been used to simulate the process, showing that for small ripples the problem is linear, and a transfer function can be defined. For amplitudes and frequency combinations above the linear threshold it is still possible to build an effective model. By only simulating a limited number of fixed amplitude transfer functions in the nonlinear regime, a semianalytical map can be constructed in order to instantly predict the spill frequency response to a given input current modulation. Both the full MADX model and the semianalytical one have been validated using data from dedicated measurements. A satisfying agreement has been achieved for almost all the experimentally injected ripples up to $200 \mathrm{~Hz}$, the only problems being related to a very low intensity of the spill signal. Operational data was also used to validate the developed model, showing difficulties to obtain a precise estimation of the $50 \mathrm{~Hz}$ harmonics on the input current. To overcome this, the model is validated using a continuous transfer function approach. A method for removing the measurement noise based on the theoretical transfer function of the measurement chain has been used, leading to a good agreement with simulation. A level of uncertainty on the operational data still remains, due to the difficulties in measuring the actual ripples component on the magnets, and the presence of other possible tune modulating effects which are not taken into account in the model. A Henon map-based model of the frequency transfer has also been developed, showing a very good agreement with the MADX one. The model presents great advantages on the simulation times and it has been used for a scan of the virtual sextupole strength and chromaticity. The results of the scan show that further attenuation of the current ripples (e.g., up to a factor 2 on the $50 \mathrm{~Hz}$ ) would be possible for smaller sextupole strength and higher chromaticity.

\section{OUTLOOK}

Future measurement campaigns will play an important role for further validating and improving the developed models. First, the issues on the measured operational current will need to be investigated in detail, with the goal of additionally validating the model and precisely estimating the discrete frequency components on the magnets. The remaining open points on the operational data, as the effect of the servo-spill systems and additional high frequency harmonics, will also be addressed. The predictions made by the Henon-map model for the sextupole strength and chromaticity scan will be tested with measurements, in order to confirm the expected improvements. A detailed study of other implications related to this change of extraction parameters will also be pursued and included in the scan. For instance, changes in the losses at the electrostatic septum, dumped intensity, spiral step, distance from the third order resonance line, or fulfillment of the Hardt condition are all important effects that will constrain the final choice of optimal chromaticity and sextupole strength pair. Concerning the injection of a dominant high frequency ripple as an alternative spill-quality improvement technique [31], the MADX simulation has confirmed its potential, but further studies will be needed to understand its compatibility with the experiment requirements. Finally, this work opens the possibility to have an online prediction of the effect of a current variation on the spill, and hence help the design and operation of slow extraction systems. For instance, this will be fundamental for the SPS to guarantee the high-quality spill demands from the North Area experiments, and preserve the SPS world leading quality of its fixed target facilities. 


\section{ACKNOWLEDGMENTS}

The authors gratefully acknowledge M. Cerqueira Bastos and M. Martino for the useful insights and help on the current measurement system of the main quadrupoles of the SPS. We also thank the SPS operators and everyone that helped to successfully carry out the measurements in the busy preshutdown months. Finally, we are grateful to the ENUBET project and collaboration, which made this project possible in the first place. This project has received funding from the European Union's Horizon 2020 Research and Innovation program under Grant Agreement No. 681647.

[1] V. Kain, CERN-SPS slow extraction overview (2017), Slow Extraction Workshop 2017, CERN, Geneva, Switzerland, https://indico.cern.ch/event/639766/ contributions/2750883/.

[2] V. Kain, F. M. Velotti, M. A. Fraser, B. Goddard, J. Prieto, L. S. Stoel, and M. Pari, Resonant slow extraction with constant optics for improved separatrix control at the extraction septum, Phys. Rev. Accel. Beams 22, 101001 (2019).

[3] M. G. Pullia et al., Betatron core driven slow extraction at CNAO and MedAustron, in Proceedings of the International Particle Accelerator Conference (IPAC'16), Busan, Korea, 2016, International Particle Accelerator Conference No. 7 (JACoW Publishing, Geneva, 2016), pp. 1330-1333, https://doi.org/10.18429/JACoWIPAC2016-TUPMR037.

[4] R. Muto et al., Current status of slow extraction from J-PARC main ring, in Proceedings of the 10th International Particle Accelerator Conference (IPAC'19), Melbourne, Australia, 2019, International Particle Accelerator Conference No. 10 (JACoW Publishing, Geneva, 2019), pp. 2311-2314, https://doi.org/10.18429/JACoWIPAC2019-WEPMP007.

[5] C. Moore, D. E. Johnson, J. Johnstone, T. Kobilarcik, and C. T. Murphy, Slow extraction from the Fermilab main injector, in PACS2001, Proceedings of the 2001 Particle Accelerator Conference (Cat. No. 01CH37268) (2001), Vol. 2, pp. 1559-1561.

[6] V. Nagaslaev, J. Amundson, J. Johnstone, L. Michelotti, C. Park, S. Werkema, and M. Syphers, Third integer resonance slow extraction scheme for a Mu2e experiment at Fermilab, in 46th ICFA Advanced Beam Dynamics Workshop on High-Intensity and High-Brightness Hadron Beams (2010), arXiv:1207.6621.

[7] K. Brown, Slow extraction and spill control from BNL's AGS and Booster (2019), ICFA Mini-Workshop on Slow Extraction, 2019, Fermilab, Batavia, IL, https://indico.fnal .gov/event/20260/contributions/56661/.

[8] D. Ondreka, Overview of slow extraction at GSI and FAIR (2017), Slow Extraction Workshop 2017, CERN, Geneva, Switzerland, https:/indico.cern.ch/event/639766/ contributions/2750884/.

[9] C. Krantz et al., Slow extraction techniques at the Marburg Ion-Beam Therapy Centre, in Proceedings of the 9th
International Particle Accelerator Conference (IPAC'18), Vancouver, BC, Canada, 2018, International Particle Accelerator Conference No. 9 (JACoW Publishing, Geneva, 2018), pp. 1084-1086, https://doi.org/10.18429/ JACoW-IPAC2018-TUPAL036.

[10] K. Noda, Overview of medical slow extraction machines in Japan (2019), ICFA Mini-Workshop on Slow Extraction, 2019, Fermilab, Batavia, IL, https://indico.fnal.gov/event/ 20260/contributions/56663/.

[11] F. Velotti et al., Characterization of SPS slow extraction spill quality degradation, in Proceedings of the 10th International Particle Accelerator Conference (IPAC'19), Melbourne, Australia, 2019, International Particle Accelerator Conference No. 10 (JACoW Publishing, Geneva, 2019), pp. 2403-2405, https://doi.org/10.18429/JACoWIPAC2019-WEPMP034.

[12] V. Kain, K. Cornelis, and E. Effinger, New spill control for the slow extraction in the multicycling SPS, in Proceedings of the International Particle Accelerator Conference (IPAC'16), Busan, Korea, 2016, International Particle Accelerator Conference No. 7 (JACoW Publishing, Geneva, 2016), pp. 1371-1373, https://doi.org/10.18429/ JACoW-IPAC2016-TUPMR051.

[13] M. Gyr, Proposal for a new servo-spill system: Power requirements for different configurations, CERN Technical Report No. CERN-SL-95-103-BT, Geneva, 1995.

[14] J. Wenninger, SPS Spill Quality, CERN Technical Report No. AB-Note-2004-0YY OP, Geneva, 2003.

[15] L. Badano, M. Benedikt, P. J. Bryant, M. Crescenti, P. Holy, A. T. Maier, M. Pullia, S. Rossi, and P. Knaus (CERN-TERA Foundation-MedAustron Oncology-2000 Collaboration), Proton-Ion Medical Machine Study (PIMMS) (1999), p. 1, https://cds.cern.ch/record/385378.

[16] V. Rodel, La dynamique de l'extraction lente du synchrotron protons de $400 \mathrm{GeV}$ du CERN en vue d'un asservissement, Ph.D. thesis, Ecole Polytechnique Federale del Lausanne, Lausanne, 1983.

[17] M. Gyr, Low frequency fluctuations of spill rates during slow resonant extraction, CERN Technical Report No. CERN-SPS-89-21-ABT, Geneva, 1989.

[18] F. Velotti et al., Observations of SPS slow-extracted spill quality degradation and possible improvements, in Proceedings of the 9th International Particle Accelerator Conference (IPAC'18), Vancouver, BC, Canada, 2018, International Particle Accelerator Conference No. 9 (JACoW Publishing, Geneva, 2018), pp. 761-764, https:// doi.org/10.18429/JACoW-IPAC2018-TUPAF035.

[19] J. Prieto, M. Fraser, B. Goddard, V. Kain, L. Stoel, and F. Velotti, Beam dynamics simulations of the effect of power converter ripple on slow extraction at the CERN SPS, in Proceedings of the 9th International Particle Accelerator Conference (IPAC'18), Vancouver, BC, Canada, 2018, International Particle Accelerator Conference No. 9 (JACoW Publishing, Geneva, 2018), pp. 818-821, https:// doi.org/10.18429/JACoW-IPAC2018-TUPAF050.

[20] K. Okamura et al., A consideration on the transfer function between RQ field and slow extraction spill in the main ring of J-PARC, in Proceedings of the 10th International Particle Accelerator Conference (IPAC'19), Melbourne, Australia, 2019, International Particle Accelerator 
Conference No. 10 (JACoW Publishing, Geneva, 2019), pp. 2315-2317, https://doi.org/10.18429/JACoWIPAC2019-WEPMP008.

[21] D. Naito, Y. Kurimoto, R. Muto, T. Kimura, K. Okamura, T. Shimogawa, and M. Tomizawa, Real-time correction of betatron tune ripples on a slowly extracted beam, Phys. Rev. Accel. Beams 22, 072802 (2019).

[22] S. Sorge, P. Forck, and R. Singh, Measurements and simulations of the spill quality of slowly extracted beams from the SIS-18 synchrotron, J. Phys. Conf. Ser. 1067, 052003 (2018).

[23] R. Singh, P. Forck, and S. Sorge, Smoothing of the slowly extracted coasting beam from a synchrotron, arXiv: 1904.09195.

[24] M. Pari, M. Fraser, B. Goddard, V. Kain, L. Stoel, and F. Velotti, Model and measurements of CERN-SPS slow extraction spill reshaping-The burst mode slow extraction, in Proceedings of the 10th International Particle Accelerator Conference (IPAC'19), Melbourne, Australia, 2019, International Particle Accelerator Conference No. 10 (JACoW Publishing, Geneva, 2019), pp. 2406-2409, https://doi.org/10.18429/JACoWIPAC2019-WEPMP035.

[25] S. van der Meer, Stochastic extraction, a low-ripple version of resonant extraction, CERN Technical Report No. CERN-PS-AA-78-6, Geneva, 1978.

[26] M. Gyr, Slow extraction at $400 \mathrm{GeV}-\mathrm{c}$ with stochastic rf noise, CERN Technical Reports No. CERN-SPS-AOPMG-jf. No. CERN-SPS-Improvement-Report-179, and No. CERN-SPS-Impr-Rep-80-179, Geneva, 1978.

[27] R. Cappi and C. Steinbach, Low frequency duty factor improvement for the CERN ps slow extraction using rf phase displacement techniques, IEEE Trans. Nucl. Sci. 28, 2806 (1981).

[28] L. Falbo, E. Bressi, and C. Priano, Empty sweeping bucket for slow extraction, in Proceedings of the 9th International Particle Accelerator Conference (IPAC'18), Vancouver, $B C$, Canada, 2018, International Particle Accelerator Conference No. 9 (JACoW Publishing, Geneva, 2018), pp. 676-678, https://doi.org/10.18429/JACoW-IPAC2018TUPAF010.

[29] A. D. Franco et al., Slow extraction optimization at the MedAustron Ion Therapy Center: Implementation of front end acceleration and rf knock out, in Proceedings of the 9th International Particle Accelerator Conference (IPAC'18), Vancouver, BC, Canada, 2018, International Particle Accelerator Conference No. 9 (JACoW Publishing, Geneva, 2018), pp. 453-456, https://doi.org/10.18429/ JACoW-IPAC2018-MOPML025.
[30] R. Singh, P. Forck, P. Boutachkov, S. Sorge, and H. Welker, Slow extraction spill characterization from micro to millisecond scale, J. Phys. Conf. Ser. 1067, 072002 (2018).

[31] R. Singh, P. Forck, and S. Sorge, Reducing fluctuations in slow-extraction beam spill using transit-time-dependent tune modulation, Phys. Rev. Applied 13, 044076 (2020).

[32] H. Grote, G. Roy, F. Schmidt, and L. Deniau, The MAD-X Program-User's Reference Manual, CERN, http://madx .web.cern.ch $/ \mathrm{madx} /$.

[33] F. M. Velotti, Higher brightness beams from the SPS for the HL-LHC era, Ph.D. thesis, Ecole Polytechnique Federale del Lausanne, Lausanne, 2017.

[34] M. A. Fraser, B. Goddard, V. Kain, M. Pari, F. M. Velotti, L. S. Stoel, and M. Benedikt, Demonstration of slow extraction loss reduction with the application of octupoles at the CERN Super Proton Synchrotron, Phys. Rev. Accel. Beams 22, 123501 (2019).

[35] F. M. Velotti, L. S. Esposito, M. A. Fraser, V. Kain, S. Gilardoni, B. Goddard, M. Pari, J. Prieto, R. Rossi, W. Scandale, L. S. Stoel, F. Galluccio, M. Garattini, and Y. Gavrikov, Septum shadowing by means of a bent crystal to reduce slow extraction beam loss, Phys. Rev. Accel. Beams 22, 093502 (2019).

[36] J. Laskar, The chaotic motion of the solar system: A numerical estimate of the size of the chaotic zones, Icarus 88, 266 (1990).

[37] J. Laskar, C. Froeschl, and A. Celletti, The measure of chaos by the numerical analysis of the fundamental frequencies. application to the standard mapping, Physica (Amsterdam) 56D, 253 (1992).

[38] M. Hénon, A two-dimensional mapping with a strange attractor, Commun. Math. Phys. 50, 69 (1976).

[39] D. Jacquet, R. Gorbonosov, G. Kruk, and P. Mira, LSAThe high level application software of the LHC and its performance during the first 3 years of operation, in Proceedings of the 14th International Conference on Accelerator and Large Experimental Physics Control Systems, (ICALEPCS'14), San Francisco, CA, 2013, International Particle Accelerator Conference No. 10 (JACoW Publishing, Geneva, Switzerland, 2013), pp. 12011204.

[40] The model and measurements have been carried out by Miguel Cerqueira Bastos, Michele Martino et al. at CERN.

[41] M. Pari, Study and development of SPS slow extraction schemes and focusing of secondary particles for the ENUBET monitored neutrino beam, Ph.D. thesis, Universitá degli Studi Di Padova, Dipartimento di Fisica e Astronomia G. Galilei, Padova, 2021 (to be published). 